138 BRONCHOPULMONARY DYSPLASIA (BPD) IN AN EXTREMELY LOW BIRTH WEIGHT (ELBW) NEWBORN CASE REPORT

${ }^{1}$ Katarina Pavičić Klancir*, ${ }^{2}$ Ivan Pavić, ${ }^{2}$ Maja Bosanac, ${ }^{1}$ Anita Pavičić Bošnjak, ${ }^{1}$ Tena Čižmešija, ${ }^{1}$ Ana Šimecki Butajla, ${ }^{1}$ Ivan Zovko. ${ }^{1}$ Clinical Hospital Sveti Duh; ${ }^{2}$ Children's Hospital Zagreb

\subsection{6/archdischild-2021-europaediatrics. 138}

Bronchopulmonary dysplasia (BPD) or chronic pulmonary disease is a recognized complication of prematurity which impacts different organ systems, most significantly lung function and neurocognitive development. Classic BPD is defined as the need for supplementary oxygen at the age of 28 days or the need for $30 \%$ or more supplementary oxygen and/or positive pressure ventilation at the corrected age of 36 weeks in newborns born before 32 weeks gestation. There are many reported treatment modalities (systemic and inhaled corticosteroid therapy, inhaled nitric oxide, vitamin A, caffeine, diuretics and bronchodilators), however in all large meta-analyses reported to date none of the modalities showed a substantially better efficacy in comparison to the others.

We are presenting a case of a premature newborn born to a 40-year old mother. This was a second pregnancy, which was uncomplicated until the $23 \mathrm{rd}$ week of gestation when premature prelabour rupture of membranes occurred, after which the mother received dexamethasone prophylaxis. At 26 weeks and 6 days of gestation a male premature newborn was born by spontaneous vaginal delivery, Apgar scores were 4, 5 and 7 at 1,5 and 10 minutes of life respectively, birth weight was 920 grams, body length $35 \mathrm{~cm}$. After primary resuscitation and stabilization in the delivery room he was transferred to the NICU, positive pressure ventilation was commenced and he received surfactant. He was extubated on day 8 of life, however even after numerous attempts at treatment (including low dose systemic and inhaled corticosteroid therapy, aminophylline, caffeine) it was not possible to reduce the need for additional oxygen, which, in combination with typical chest $\mathrm{X}$-ray changes, led to confirming the diagnosis of BPD. Additionally, he developed retinopathy or prematurity (ROP), which was treated by intravitreous application of anti-VEGF with very good response. On $2 \mathrm{D}$ brain ultrasound a grade 2 intarventricular hemorrhage (IVH) was seen, that remained stable during follow up and the MRI at 40 weeks corrected gestational age was normal. At 3 months of age, considering persisting need for supplementary oxygen, therapy with oral theophylline and inhaled fluticasone was commenced, after which the need for supplementary oxygen reduced to 0.1-0.2 $\mathrm{L} / \mathrm{min}$ and the lung auscultatory findings were improved at discharge. Outpatient follow-up was continued - including pulmonologist, neuropediatrician, rehabilitation specialist, speech therapist. At 9 months of age (6 months corrected age) the infant doesn't require supplementary oxygen and has satisfactory weight gain. Further multidisciplinary follow-up of growth and development is essential, as well as lung function monitoring, which will determine management of therapy with theophylline and fluticasone.

Although there is no specific diagnostic test or therapy for children with BPD, careful selection of therapeutic modalities as well as methodical medication adjustment can significantly contribute to prevention, elimination or significant reduction of symptoms in children with BPD.

\section{NEONATALLISTERIOSIS - A CASE REPORT}

Lorita Mihovilović Prajz ${ }^{*}$, Lucija Debeljak Poslek, Tatjana Sotonica Pirija, Saša Baranjac, Tvrtko Kovačević. KBC Sestre milosrdnice

10.1136/archdischild-2021-europaediatrics. 139

Neonatal listeriosis is a rare but severe infectious disease caused by the gram-positive bacterium Listeria monocytogenes with a mortality rate of almost 30\%. Pregnant women are mostly infected with Listeria by consuming contaminated food but cases of listeriosis after direct contact with an infected animal have been reported. Neonatal listeriosis has two clinical presentations, early and late-onset. Early onset listeriosis is caused by transplacental transmission or ascending infection with consequent chorioamnionitis, premature rupture of membranes, meconium amniotic fluid and neonatal sepsis, often with the presence of numerous granulomas mostly in spleen and liver of the infant. Late onset listeriosis is caused by an infection transmitted to the newborn when it passes through the birth canal or is, less likely, a nasocomial infection. Late onset listeriosis is manifested as meningoencephalitis between the 1 st and 2 nd week of life and is associated with a better prognosis.

We report a case of a male newborn born in the 38th week of gestation from a febrile mother, born in meconium amniotic fluid, which was admitted to the neonate intensive care unit in the first hours of life due to development of respiratory distress syndrome and signs of early neonatal sepsis. After obtaining microbiological samples, empirical therapy with ampicillin and gentamicin was introduced. Listeria monocytogenes was isolated from skin swabs and gastric aspirate of the newborn, blood culture as well as urine and cerebrospinal fluid culture came sterile; cerebrospinal fluid PCR was also negative for Listeria DNA. We did not radiologically verify presence of granulomas in any organ. Following this result, cervical and vaginal swabs were obtained from the mother and also revealed presence of L. monocytogenes. In the further course, the doses of antibiotics were adjusted and the newborn was afebrile, control microbiological samples were sterile and he was discharged after 16 days. Reviewing medical records, it was noticed that the 1 st child of this mother also had a perinatal infection where the causative agent was not isolated, and given the anamnestic data that the family is engaged in sheep breeding, it is possible that the background was also unrecognized L. monocytogenes infection. In conclusion, a blood culture sample and cervical swab should be done to any pregnant woman with an unclear febrile condition, as Listeria monocytogenes is a common unrecognized cause of miscarriages and stillbirths, and early treatment is necessary to prevent vertical transmission and thus severe complications and fatalities in newborns.

\section{PERSONALIZED GROWTH TRAJECTORIES - A BEDSIDE TOOL FOR MONITORING GROWTH OF PRETERM INFANTS}

Edi Paleka Bosak*, Gabrijela Perić Marković, Vinko Vrdoljak, Rebeka Ribičić, Snježana Gverić-Ahmetašević. Klinički bolnički centar 'Sestre Milosrdnice'

10.1136/archdischild-2021-europaediatrics. 140

Fenton growth charts are commonly used to monitor postnatal growth of preterm infants. New individualized growth chart 
concept arises from 4 phases of growth: phase of intrauterine growth, phase of completed postnatal adjustment in first 3 weeks of life forming a percentile below birth percentile, phase of stable growth targeting birth weight percentile at 42 $+0 / 7$ week, according to Fenton chart and phase of preterm infant growth after term age monitored with WHO growth charts. The aim of this study is to present a new approach for personalized postnatal growth trajectories for preterm infants and to compare them with growth percentiles of our patients. This concept still needs to be validate in a larger sample size. Data from 39 preterm infants gestational age (GA) 29-33+6/7 weeks and birth weight $(\mathrm{BW})<2000 \mathrm{~g}$ were collected. Retrospective, for every infant, a new personalized growth chart was drawn using online calculator (https://www. growthcalculator.org/) and was compared with existing growth trajectory measured from birth to discharge. The difference between measured (MW) and predicted weights (PW) of our patients were calculated and analyzed, together with their characteristics, comorbidities, treatment and nutrition. Preliminary results show that the most of them $(\mathrm{N}=22)$ deviated less than $5 \%$ of ideal weight (PW) and there were no differences between sexes. Statistically significant deviation in median for MW and PW was noticed in a preterm infants GA $>32$ weeks. Remaining results will be presented after analysing data of total number of patients collected from hospital records from period 2018 to 2020. Adjusting nutrition in the earliest phase of postnatal life towards an ideal personalized growth trajectory, can have an optimal impact in growth, body composition and neurodevelopment of the infant and can reduce the risk of some early onset adult metabolic and cardiovascular diseases. Therefore, it is necessary to create more precise tools in monitoring growth of preterm infants, such as personalized growth trajectories.

\section{KAPOSIFORM HAEMANGIOENDOTHELIOMA WITH KASABACH-MERRITT PHENOMEN IN A NEWBORN: A CASE REPORT}

Karmen Kondža*, Ivančica Škarić. Children's Hospital Zagreb, Zagreb, Croatia

10.1136/archdischild-2021-europaediatrics. 141

Kaposiform haemangioendothelioma ( KHE) is a rare vascular tumor and has high mortality rate in newborns when associated with Kasabach-Merritt syndrome ( KMS) due to consumptive coagulopathy.

Methods Female newborn, GA 30 w, BM 2220g, due to the threatening asphyxia, born by S.C., with respiratory distress syndrome (RDS) and extremly massive soft tissue purple tumor with swollen erythematous, inhomogenous skin. The tumor was spread over lower abdominal wall, vulva, gluteal region, whole right tight and upper part of the left tight. The baby was anemic ( $\mathrm{Hb} 97 \mathrm{~g} / \mathrm{l})$, trombocytopenic ( 8x109/L) with consumptive coagulopathy (immeasurable fibrinogen). RDS was treated with exogenous surfactant and mechanical ventilation. As soon as possible she received packed red blood cell transfusion, platelet transfusion, fibronogen concentrate, INN-eptacog alfa (activated). On the ultrasound of the abdomen paralytic ileus and hemorrhagic effusions were found. Doppller ultrasound of the tumor expressed heterogenous echogenicity and hypervascular pattern. Hour by hour the tumor grew larger. In spite of all intensive treatment baby developed multiorgan failure (MOF) and died in the of 18 hours.

Results Patohistologically, the tumor consists of irregular, predominantly small and slit-like vascular spaces lined with spindle endothelial cells which sometimes form nodular structure. On immunohistochemistry the spindle cells had positive reaction for CD31, CD34, D2-40 and negative reaction for GLUT-1. The finding corresponds to KHE.

Conclusions We report a premature born neonate with a huge KHE associated with fulminant form of KMS and developed consumptive coagulopathy resulting in multiorgan failure and death within 18 hours.

\section{Paediatric Allergology and Clinical Immunology}

\section{DRUG-INDUCED ENTEROCOLITIS SYNDROME (DIES) IN 6-YEAR OLD GIRL}

Renata Vrsalović*, Iva Škorić, Iva Mihatov Štefanović, Biserka Čičak. Department of Pediatrics, Sestre milosrdnice University Hospital Center, Zagreb, Croatia

\subsection{6/archdischild-2021-europaediatrics. 142}

Drug-induced enterocolitis syndrome (DIES) is an uncommon, non-IgE-mediated drug hypersensitivity reaction that can be severe and potentially life-threatening disease. Because of the clinical resemblance with enterocolitis syndrome induced by food proteins (FPIES), DIES is also called 'FPIES-like' reaction and similar diagnostic criteria are proposed. We report the case of a 6-year old girl who was admitted to our Department for an oral challenge test with amoxicillin (AMX).

She was under the supervision of pulmonologist for recurrent wheezing episodes. During the outpatient- follow up mother reported multiple reactions after the ingestion of amoxicillin +/- clavulanic acid. After the first administration of oral suspension girl developed an erythematous skin rash, abdominal pain followed with an acute episode of repetitive vomiting.

Next two administrations were followed with a short period of drowsiness, abdominal pain, repetitive vomiting and severe diarrhea 1-2 hours after drug ingestion. The symptoms spontaneously resolved within 24 hours. During the last reaction parents called an ambulance because of drowsiness and poor general condition after severe vomiting and diarrhea, and girl was treated shortly with intravenous infusion.

In the allergy study performed, specific IgE to amoxicillin was negative as well as basophil activation test for Augmentin ${ }^{\circledR}$.

Consecutively, we performed an open 3-step graded oral provocation test with AMX in a hospital setting according to the published guidelines.

Approximately 3 hours after receiving the first dose $(5 \mathrm{mg})$ and 1 hour after the second dose $(50 \mathrm{mg})$, she developed severe abdominal pain, nausea and repetitive vomiting, and two hours later she became pale and developed severe diarrhea. During the reaction, she had no cutaneous or respiratory symptoms, and she remained hemodynamically stable. A blood test obtained 1 hour after the onset of the reaction showed normal complete blood cell count. She was parenterally hydrated. Approximately 5-6 hours after onset of symptoms she showed progressive improvement to a complete recovery. 